\title{
Generic Algorithm Implementation of Approximation Algorithm using Simulated Annealing (SA)
}

\author{
Diptam Dutta \\ Computer Science \& Engineering \\ Heritage Institute of Technology \\ drozendutta@gmail.com
}

\begin{abstract}
This paper describes a complementary mechanism that attempts to learn the structure of the search space over multiple runs of SA on a given problem[29] (Best fit Problem \& First Fit Decreasing). Specifically, we introduce a mechanism that attempts to predict how (UN) promising a SA runs is likely to be, based on probability distributions that are "learned" over multiple runs. The distributions, which are built at different checkpoints, each corresponding to a different value of the temperature ('temperature' is a variable which decrements its value at each step-as SA has a great relation with physics, the variable is termed in this manner) parameter used in the procedure, approximate the cost reductions that one can expect if the SA run is continued below these temperatures.

Simulated annealing is a method of finding optimal values numerically. It chooses a new point, and (for optimization) all uphill points are accepted while some downhill points are accepted depending on probabilistic criteria. For certain problems, simulated annealing may be more efficient than exhaustive enumeration - provided that the goal is to find an acceptably good solution in a fixed amount of time, rather than the best possible solution.
\end{abstract}

\section{Keyword}

One bin packing, multiple bins packing, simulated annealing, best fit problem, first fit decreasing, meta- heuristics, constraints (parameters).

\section{INTRODUCTION}

Simulated Annealing is a local search method based on local optimization. In this method each trial solution in the solution space has a cost, and the objective is to find a feasible solution of least cost. The method is iterative. In each cycle we try to move from the current trial solution $S$ to a neighboring point $S^{\prime}$ in the solution space in an effort to find a better trial solution.

Let us assume that the problem is a minimization problem. If $\operatorname{cost}\left(S^{\prime}\right)<\operatorname{cost}(\mathrm{S})$, $\mathrm{S}^{\prime}$ becomes the new trial solution; the move from $S$ to $S^{\prime}$ is then called a downhill move. If $\operatorname{cost}\left(S^{\prime}\right)>$ $\operatorname{cost}(\mathrm{S}), \mathrm{S}^{\prime}$ becomes the new trial solution with probability $\mathrm{p}=$ $\exp (-\Delta /$ temp), where temp is a parameter known as the temperature and $\Delta=\operatorname{cost}\left(\mathrm{S}^{\prime}\right)-\operatorname{cost}(\mathrm{S})$; $\mathrm{S}$ is retained as the trial solution with probability (1-p). Thus $S^{\prime}$ can become the new trial solution even when its cost is higher than the cost of the current trial solution $\mathrm{S}$; this kind of move from $\mathrm{S}$ to $\mathrm{S}$ ' is called an uphill move. This deliberate choice of an inferior trial solution with a non-zero probability helps to ensure that the procedure does not get trapped in a local minimum. By slowly reducing the temperature, the probability $p$ is reduced in the course of the iteration as better trial solutions are found.
Bin packing [1] problem solves the packing of objects of different volumes into a finite number of bins of capacity $\mathrm{V}$ in a way that minimizes the number of bins used. The approximation algorithm is applied on Multiple Bin Packing Problem in such a way that the algorithm produces the minimum number of bin used as a result.

\section{LITERATURE REVIEW}

\subsection{Bin Packing Problem}

The bin packing problem asks for the minimum number of identical bins of capacity $\mathrm{C}$ needed to store a finite collection of weights $w_{1}, w_{2}, w_{3} \ldots w_{n}$ so that no bin has weights stored in it whose sum exceeds the bin's capacity. Traditionally the capacity $\mathrm{C}$ is chosen to be 1 and the weights are real numbers which lie between 0 and 1 , but here, for convenience of exposition, I will consider the situation where $\mathrm{C}$ is a positive integer and the weights are positive integers which are less than the capacity.

\subsection{Simulated Annealing}

Simulated Annealing (SA) is a general-purpose search procedure that generalizes iterative improvement approaches to combinatorial optimization by sometimes accepting transitions to lower quality solutions to avoid getting trapped in local minima. SA procedures have been successfully applied to a variety of combinatorial optimization problems, including Traveling Salesman Problems, Graph Partitioning Problems , Graph Coloring Problems[20], Vehicle Routing Problems[15] , Design of Integrated Circuits, Minimum Make-span Scheduling Problems as well as other complex scheduling problems, often producing near-optimal solutions, though at the expense of intensive computational efforts. The procedures, typically requiring that the procedure be rerun (iterate) a large number of times before a near optimal solution are found. Other names of Simulated Annealing are Monte Carlo Annealing[5], Statistical Cooling[6], Probabilistic Hill Climbing[7], Stochastic Relaxation[9], Probabilistic Exchange Algorithm[8] etc.

\subsection{Problem Definition}

The problem is categorized into two phases i.e., Phase I \& Phase II

2.3.1. Phase I: The goal is to fit the different weighted objects into a single bin with the least cost function.

2.3.2. Phase II: The goal is to fit the different weighted objects into multiple bins such that minimum number of bins used. 


\subsection{Proposed Work}

2.4.1. Proposed Algorithm For Simulated Annealing:

Procedure SA

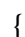

$$
\text { if }(\Delta<0)
$$

\subsection{Setting up SINGLE BIN PACKING PARAMETERS and Approximation Analysis with SIMULATED ANNEALING}

We need to initialize parameters based on ITEMLIST (total number of items) and MAXBINSIZE

Round_1 $:$ ITEMLIST $=5 ;$ MAXBINSIZE $=100$; INITIALSOLUTIONLIST (no. of objects to create an initial_Soln $)=$ ITEMLIST $* 60 \%$ REDUCEDBINSIZE $=$ MAXBINSIZE $* 50 \%$; NEIGHBOURCREATION=INITIALSOLUTIONLIST * $33 \%$

Round_2 $:$ ITEMLIST $=15 ;$ MAXBINSIZE $=300$; INITIALSOLUTIONLIST (no. of objects to create an initial_Soln $)=$ ITEMLIST $* 60 \%$ REDUCEDBINSIZE $=$ MAXBINSIZE $* 25 \%$; NEIGHBOURCREATION=INITIALSOLUTIONLIST * $33 \%$

Round 3 : ITEMLIST =75; MAXBINSIZE = 900; INITIALSOLUTIONLIST (no. of objects to create an initial_Soln $)=$ ITEMLIST $* 60 \%$ REDUCEDBINSIZE $=$ MAXBINSIZE $* 15 \%$; NEIGHBOURCREATION=INITIALSOLUTIONLIST * $33 \%$

Round_4 : ITEMLIST =225; MAXBINSIZE $=2700$; INITIALSOLUTIONLIST (no. of objects to create an initial_Soln $)=$ ITEMLIST $* 60 \%$ REDUCEDBINSIZE $=$ MAXBINSIZE $* 15 \%$; NEIGHBOURCREATION=INITIALSOLUTIONLIST * $33 \%$

Round_5 $:$ ITEMLIST $=675 ;$ MAXBINSIZE $=8100$; INITIALSOLUTIONLIST (no. of objects to create an initial_Soln $)=$ ITEMLIST $* 60 \%$ REDUCEDBINSIZE $=$ MAXBINSIZE $* 10 \%$; NEIGHBOURCREATION=INITIALSOLUTIONLIST * $33 \%$

Round_6 $:$ ITEMLIST $=2025 ;$ MAXBINSIZE $=24300$; INITIALSOLUTIONLIST (no. of objects to create an initial_Soln) $=$ ITEMLIST $* 60 \%$ REDUCEDBINSIZE $=$ MAXBINSIZE $* 10 \%$; NEIGHBOURCREATION=INITIALSOLUTIONLIST * $33 \%$

Round_7 $:$ ITEMLIST $=6075 ;$ MAXBINSIZE $=72900$; INITIALSOLUTIONLIST (no. of objects to create an initial_Soln $)=$ ITEMLIST $* 60 \%$ REDUCEDBINSIZE $=$ MAXBINSIZE $* 10 \%$; NEIGHBOURCREATION=INITIALSOLUTIONLIST * $33 \%$

Round $8:$ ITEMLIST $=18225 ;$ MAXBINSIZE $=218700$; INITIALSOLUTIONLIST (no. of objects to create an initial_Soln $)=$ ITEMLIST $* 60 \%$ REDUCEDBINSIZE $=$ MAXBINSIZE $* 10 \%$; NEIGHBOURCREATION=INITIALSOLUTIONLIST * $33 \%$ 


\subsection{Setting up Multiple BIN PACKING PARAMETERS and Approximation Analysis with SIMULATED ANNEALING}

We need to initialize parameters based on MAXBINSIZE and MAXOBJNO

Round_1 : MAXOBJNO=6; MAXBINSIZE $=10$;[where $\mathrm{x}=6 ; \mathrm{y}=10$ with $6: 10=3: 5$ ratio]

For NEIGHBOURCREATION: Option 1 -> Replace All randomly generated objects

Option 2 -> Replace 50\% (3 objects at a time )

Option $3 \rightarrow$ Replace $25 \%$ ( 1 or 2 objects at a time based on floor or ceil values);

Round_2: MAXOBJNO=12; MAXBINSIZE $=20$; [where $2 \mathrm{x}=12 ; 2 \mathrm{y}=20$ with $12: 20=3: 5$ ratio]

For NEIGHBOURCREATION: Option 1 -> Replace All randomly generated objects

Option $2 \rightarrow$ Replace 50\% (6 objects at a time)

Option 3 -> Replace $25 \%$ ( 3 objects at a time);

Round_3: MAXOBJNO=24; MAXBINSIZE $=40$; [where2 $\mathrm{x}=24 ; 2 \mathrm{y}=40$ with $24: 40=3: 5$ ratio]

For NEIGHBOURCREATION: Option 1 -> Replace All randomly generated objects

Option $2 \rightarrow$ Replace $50 \%$ (12 objects at a time )

Option 3 -> Replace $25 \%$ (6 objects at a time );

Round_4 : MAXOBJNO=48; MAXBINSIZE $=80$; [where $2 \mathrm{x}=48 ; 2 \mathrm{y}=80$ with $48: 80=3: 5$ ratio]

For NEIGHBOURCREATION: Option 1 -> Replace All randomly generated objects

Option 2 -> Replace 50\% (24 objects at a time )

Option 3 -> Replace $25 \%$ (12 objects at a time );

Round_5 : MAXOBJNO=96; MAXBINSIZE $=160 ;$ [where $2 \mathrm{x}=96 ; 2 \mathrm{y}=160$ with $96: 160=3: 5$ ratio]

For NEIGHBOURCREATION: Option 1 -> Replace All randomly generated objects

Option 2 -> Replace 50\% (48 objects at a time )

Option $3 \rightarrow$ Replace $25 \%$ ( 24 objects at a time );

Round_6 : MAXOBJNO=192; MAXBINSIZE $=320$;[where $2 \mathrm{x}=192 ; 2 \mathrm{y}=320$ with $192: 320=3: 5$ ratio]

For NEIGHBOURCREATION: Option 1 -> Replace All randomly generated objects

Option 2 -> Replace 50\% (96 objects at a time )

Option 3 -> Replace $25 \%$ (48 objects at a time );

Round_7 : MAXOBJNO=384; MAXBINSIZE $=640$; [where2 $\mathrm{x}=384 ; 2 \mathrm{y}=640$ with $384: 640=3: 5$ ratio]

For NEIGHBOURCREATION: Option 1 -> Replace All randomly generated objects

Option $2 \rightarrow$ Replace 50\% (192 objects at a time )

Option 3 -> Replace $25 \%$ (96 objects at a time );

Round_8 : MAXOBJNO=768; MAXBINSIZE $=1280$; [where2 $\mathrm{x}=768 ; 2 \mathrm{y}=1280$ with $768: 1280=3: 5$ ratio]

For NEIGHBOURCREATION: Option 1 -> Replace All randomly generated objects

Option $2 \rightarrow$ Replace 50\% (384 objects at a time )

Option 3 -> Replace $25 \%$ (192 objects at a time ); 
3. RESULT ANALYSIS

3.1.For One Bin packing

\begin{tabular}{|c|c|c|c|c|c|c|c|c|c|c|c|}
\hline $\begin{array}{l}\text { Targ } \\
\text { et } \\
\text { (T): } \\
\text { To } \\
\text { reac }\end{array}$ & $\begin{array}{l}\text { Round } \\
\text { 1: } \\
\text { Trails } \\
=25\end{array}$ & $\begin{array}{l}\text { Round } \\
\text { 2: Trails } \\
=\mathbf{5 0}\end{array}$ & $\begin{array}{l}\text { Round } \\
\text { 3: Trails } \\
=100\end{array}$ & $\begin{array}{l}\text { Round } \\
\text { 4: Trails } \\
=200\end{array}$ & $\begin{array}{l}\text { Round } \\
\text { 5: Trails } \\
=400\end{array}$ & $\begin{array}{l}\text { Roun } \\
\text { d 6: } \\
\text { Trails } \\
=800\end{array}$ & $\begin{array}{l}\text { Roun } \\
\text { d 7: } \\
\text { Trails } \\
= \\
\mathbf{1 6 0 0} \\
\end{array}$ & $\begin{array}{l}\text { Roun } \\
\text { d 8: } \\
\text { Trails } \\
= \\
\mathbf{3 2 0 0} \\
\end{array}$ & $\begin{array}{l}\text { Roun } \\
\text { d 9: } \\
\text { Trails } \\
= \\
6400 \\
\end{array}$ & \multirow[t]{2}{*}{$\begin{array}{l}\text { Average } \\
\text { Bin Size } \\
\text { Reached } \\
\text { (T) }\end{array}$} & \multirow[t]{2}{*}{$\begin{array}{l}\text { Differential } \\
\text { Error:Abso } \\
\text { lute Value } \\
\text { of }\left(T^{\prime}-T\right) / T\end{array}$} \\
\hline $\begin{array}{l}\text { h } \\
\text { Max } \\
\text { Bin } \\
\text { Size }\end{array}$ & $\begin{array}{l}\text { Frzlim } \\
=5 ; \\
\text { trail } \\
\text { Limit= } \\
5\end{array}$ & $\begin{array}{l}\text { Frzlim= } \\
\text { 10; trail } \\
\text { Limit }=5\end{array}$ & $\begin{array}{l}\text { Frzlim= } \\
\text { 20; trail } \\
\text { Limit=5 }\end{array}$ & $\begin{array}{l}\text { Frzlim= } \\
\text { 40; trail } \\
\text { Limit }=5\end{array}$ & $\begin{array}{l}\text { Frzlim= } \\
\text { 80; trail } \\
\text { Limit=5 }\end{array}$ & $\begin{array}{l}\text { Frzli } \\
\text { m= } \\
\text { 160; } \\
\text { trail } \\
\text { Limit } \\
=5\end{array}$ & $\begin{array}{l}\text { Frzli } \\
\text { m= } \\
\text { 320; } \\
\text { trail } \\
\text { Limit } \\
=5\end{array}$ & $\begin{array}{l}\text { Frzli } \\
\text { m= } \\
640 ; \\
\text { trail } \\
\text { Limit } \\
=5\end{array}$ & $\begin{array}{l}\text { Frzli } \\
\text { m= } \\
\text { 1200; } \\
\text { trail } \\
\text { Limit } \\
=5\end{array}$ & & \\
\hline 100 & 95 & 99 & 82 & 100 & 72 & 98 & 100 & 91 & 99 & 93 & 0.07 \\
\hline 300 & 300 & 300 & 300 & 299 & 300 & 300 & 300 & 300 & 300 & 300 & 0 \\
\hline 900 & 844 & 897 & 899 & 900 & 884 & 900 & 900 & 900 & 900 & 896 & 0.004444 \\
\hline 2700 & 2684 & 2696 & 2576 & 2698 & 2694 & 2696 & 2699 & 2700 & 2700 & 2683 & 0.006296 \\
\hline 8100 & 7927 & 8063 & 8024 & 8097 & 8093 & 8100 & 8100 & 8099 & 8100 & 8067 & 0.004074 \\
\hline $\begin{array}{l}2430 \\
0 \\
\end{array}$ & 24012 & 24154 & 24219 & 24187 & 24199 & 24230 & 24259 & 24279 & 24178 & 24191 & 0.004485 \\
\hline $\begin{array}{l}7290 \\
0 \\
\end{array}$ & 70912 & 71861 & 72454 & 72865 & 72756 & 72888 & 72565 & 72701 & 72876 & 72431 & 0.006433 \\
\hline $\begin{array}{l}2187 \\
00\end{array}$ & 217772 & 216438 & 215792 & 216754 & 214778 & $\begin{array}{l}21786 \\
5 \\
\end{array}$ & 21720 & $\begin{array}{l}21682 \\
0 \\
\end{array}$ & $\begin{array}{l}21382 \\
0 \\
\end{array}$ & 21637 & 0.010640 \\
\hline
\end{tabular}




\subsection{Analysis of Approximation Algorithms of Different Rounds:}

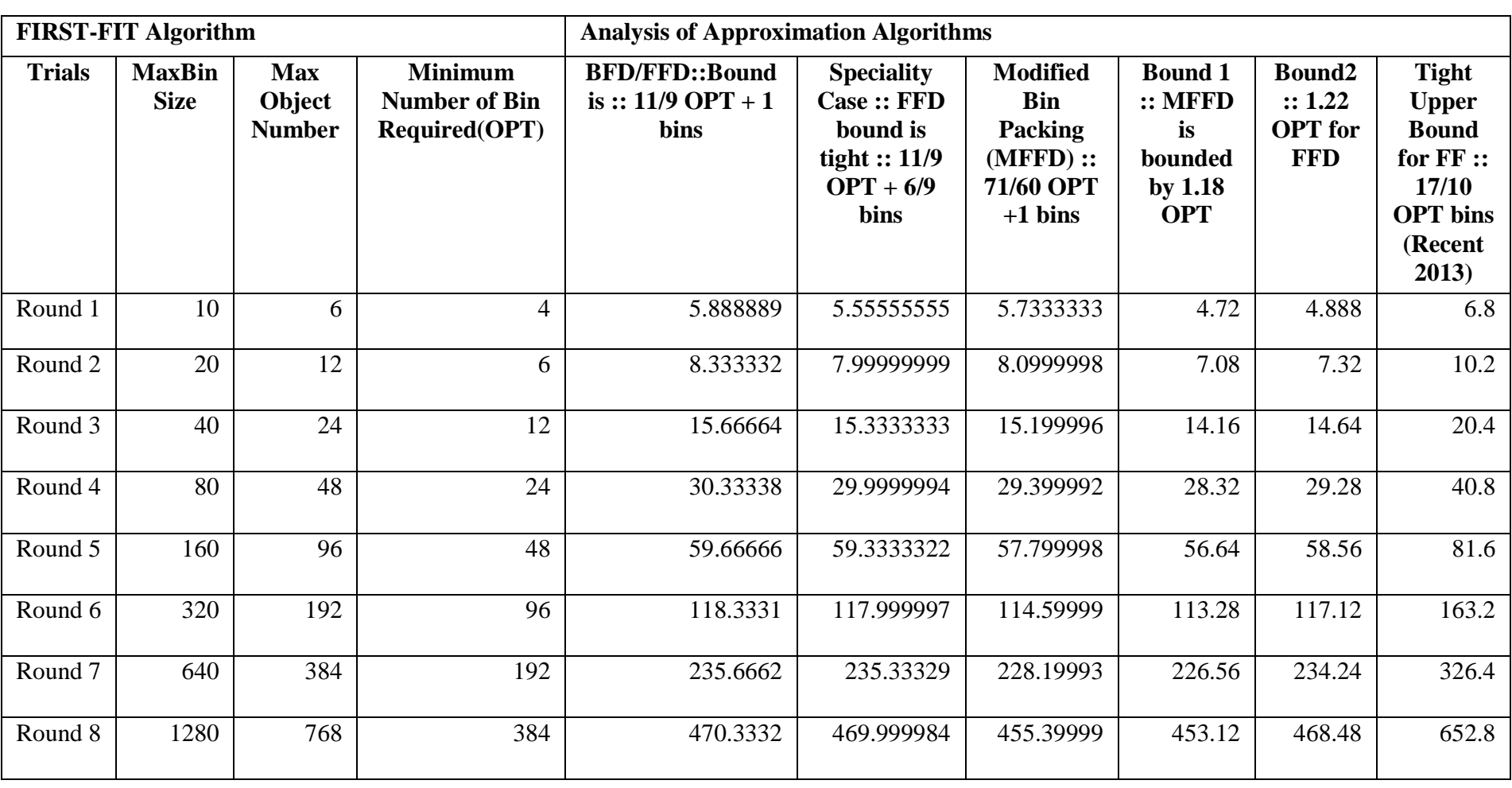




\subsection{For Multiple Bin packing:}

Table 1: Resultant Data

\begin{tabular}{|l|l|l|l|}
\hline Trials & $\begin{array}{l}\text { Max } \\
\text { Bin } \\
\text { Size }\end{array}$ & $\begin{array}{l}\text { Max } \\
\text { Object } \\
\text { Number }\end{array}$ & $\begin{array}{l}\text { Minimum } \\
\text { number } \\
\text { of Bin } \\
\text { Required } \\
\text { (OPT) }\end{array}$ \\
\hline Round 1 & 14 & 4 & 3 \\
\hline Round 2 & 28 & 8 & 4 \\
\hline Round 3 & 56 & 16 & 8 \\
\hline Round 4 & 112 & 32 & 16 \\
\hline Round 5 & 224 & 64 & 32 \\
\hline Round 6 & 448 & 128 & 64 \\
\hline Round 7 & 896 & 256 & 128 \\
\hline Round 8 & 1792 & 512 & 256 \\
\hline
\end{tabular}

\begin{tabular}{|l|l|l|l|}
\hline Trials & $\begin{array}{l}\text { Max } \\
\text { Bin } \\
\text { Size }\end{array}$ & $\begin{array}{l}\text { Max } \\
\text { Object } \\
\text { Number }\end{array}$ & $\begin{array}{l}\text { Minimum } \\
\text { number } \\
\text { of Bin } \\
\text { Required } \\
\text { (OPT) }\end{array}$ \\
\hline Round 1 & 14 & 4 & 5.1 \\
\hline Round 2 & 28 & 8 & 6.8 \\
\hline Round 3 & 56 & 16 & 13.6 \\
\hline Round 4 & 112 & 32 & 27.2 \\
\hline Round 5 & 224 & 64 & 54.4 \\
\hline Round 6 & 448 & 128 & 108.8 \\
\hline Round 7 & 896 & 256 & 217.6 \\
\hline Round 8 & 1792 & 512 & 435.2 \\
\hline
\end{tabular}

Maximum Object

Number \&

Minimum Number

of Bin required in this proposed work.

300

(1)

250

200

SERIES 2

Maximum Object

Number \&

Minimum Number

of Bin in 2013[27]

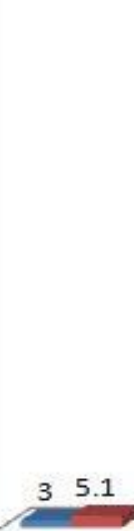

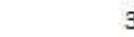

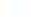

- Series1 Series2

Figure 1: Comparison plot of Resultant data. 
Table 3: Resultant Data

\begin{tabular}{|l|l|l|l|}
\hline Trials & $\begin{array}{l}\text { Max } \\
\text { Bin } \\
\text { Size }\end{array}$ & $\begin{array}{l}\text { Max } \\
\text { Object } \\
\text { Number }\end{array}$ & $\begin{array}{l}\text { Minimum } \\
\text { number } \\
\text { of Bin } \\
\text { Required } \\
\text { (OPT) }\end{array}$ \\
\hline Round 1 & 4 & 14 & 7 \\
\hline Round 2 & 8 & 28 & 15 \\
\hline Round 3 & 16 & 56 & 28 \\
\hline Round 4 & 32 & 112 & 56 \\
\hline Round 5 & 64 & 224 & 112 \\
\hline Round 6 & 128 & 448 & 224 \\
\hline Round 7 & 256 & 896 & 448 \\
\hline Round 8 & 512 & 1792 & 896 \\
\hline
\end{tabular}

\begin{tabular}{|l|l|l|l|}
\hline Trials & $\begin{array}{l}\text { Max } \\
\text { Bin } \\
\text { Size }\end{array}$ & $\begin{array}{l}\text { Max } \\
\text { Object } \\
\text { Number }\end{array}$ & $\begin{array}{l}\text { Minimum } \\
\text { number } \\
\text { of Bin } \\
\text { Required } \\
\text { (OPT) }\end{array}$ \\
\hline Round 1 & 4 & 14 & 11.9 \\
\hline Round 2 & 8 & 28 & 25.5 \\
\hline Round 3 & 16 & 56 & 47.6 \\
\hline Round 4 & 32 & 112 & 95.2 \\
\hline Raund 5 & 64 & 224 & 190.4 \\
\hline Rbund 6 & 128 & 448 & 380.8 \\
\hline Rסund 7 & 256 & 896 & 761.6 \\
\hline Rqund 8 & 512 & 1792 & 1523.2 \\
\hline
\end{tabular}

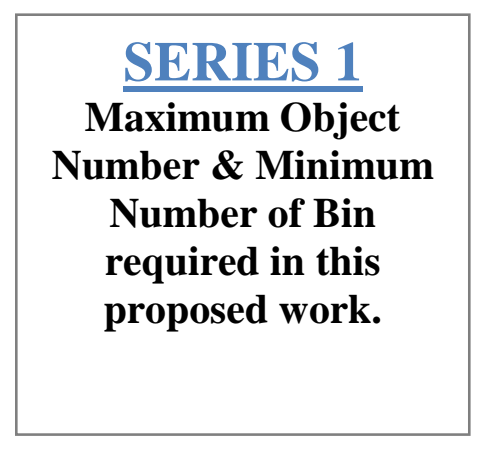

1600
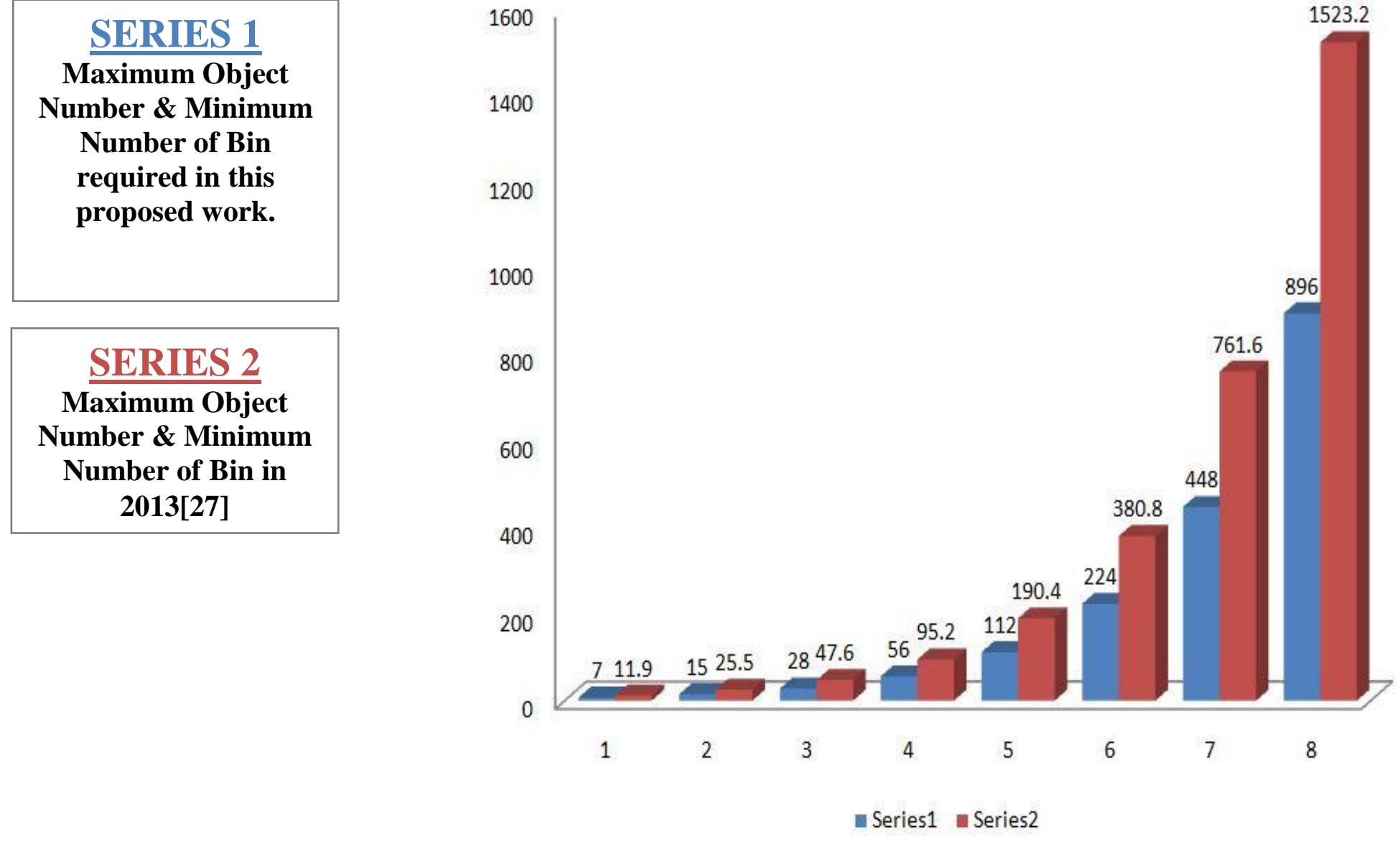

Figure 2: Comparison plot of Resultant data in a Reversed Ratio. 


\section{CONCLUSIONS}

This work has been accomplished on a single bin of variable sizes with the implementation of simulated annealing on that particular bin with least runtime complexity. Also on multiple bins of variable sizes with the implementation of simulated annealing with minimum number of bins used, got accomplished on this work. A future aspect is to implement the above problems of 1 bin packing as well as multiple bins packing in a 2-dimensional pattern.

\section{REFERENCES}

[1] Assmann, S. and D. Johnson, D. Kleitman, J. Leung, On a dual version of the one-dimensional bin packing problem, J. Algorithms 5 (1984) 502-525.

[2] Baker, B., A new proof for the first-fit decreasing binpacking algorithm, J. Algorithms 6 (1985) 49-70.

[3] Baker, B. and E. Coffman, Jr., A tight asymptotic bound for next-fit-decreasing bin packing, SIAM J. Alg. Disc. Math., 2 (1981) 147-152.

[4] Bentley, J. and D. Johnson, F. Leighton, C. McGeoch, L. McGeoch, Some unexpected expected behavior results for bin packing., in Proceedings of the 16th Annual ACM Sym. on Theory of Computing, 1984, p. 279-288.

[5] Brucker, P., Scheduling Algorithms, Springer-Verlag, New York, 1995.1

[6] Coffman, Jr., and G. Galambos, S. Martello, and D. Vigo, Bin Packing Appoximation Algorithms: Combinatorial Analysis, in Handbook of Combinatorial Optimization, D. Du and P. Pardalos, (eds.), Kluwer, Amsterdam, 1998

[7] Coffman, Jr., and M. Garey, D. Johnson, Dynamic bin packing, SIAM J. Comput., 12 (1983) 227-258.

[8] Coffman, Jr., and M. Garey, D. Johnson, Approximation Algorithms for Bin-Packing,: An updated survey, in Algorithm Design for Computer Systems Design, G. Ausiello, M. Lucertini, and P. Serafini, (eds.), SpringerVerlag, New York, 1984, 49-106.

[9] Conway, R. and W. Maxwell, L. Miller, Theory of Scheduling, Addison-Wesley, Reading, 1967.

[10] Courcoubetis, C. and R. Weber, Necessary and sufficient conditions for the stability of a bin

[11] packing system, J. Appl. Prob., 23 (1986) 989-999.

[12] Csirik, J., The parametric behavior of the first-fit decreasing bin packing algorithm, J. Algorithms 15 (1993) 1-28.

[13] Csirik, J. and J. Frenk, G. Galambos, A. RinnooyKan, Probabilistic analysis of algorithms for dual bin packing problems, J. Algorithms 12 (1991) 189-203.
[14] Csirik, J. and D. Johnson, Bounded space on-line bin packing; best is better than first, In Proceedings, Second Annual ACM-SIAM Symposium on Discrete Algorithms, SIAM, Philadelphia, 1991, p. 309-319.

[15] Fernandez del la Vega, W. and G. Lueker, Bin packing can be solved in $1+\varepsilon$ in linear time, Combinatorica 1 (1981) 34-355.

[16] Flexzar, k. and K. Hindi, New heuristics for onedimensional bin packing, Computers and Operations Research 29 (1902) 821-839.

[17] Floyd, S. and R. Karp, FFD bin packing for item sizes with distribution on [0, 1/2], Algorithmica, 6 (1991) 222240 .

[18] French, S., Sequencing and Scheduling, Wiley, New York, 1982.

[19] Garey, M. and R. Graham, D. Johnson, A. Yao, Resource constrained scheduling as generalized bin packing, J. Combinatorial Theory Ser. A, 21 (1976) 257-298.

[20] Garey, M., and D. Johnson, Approximation algorithms for bin packing problems-A survey, in Analysis and Design of Algorithms in Combinatorial Optimization, G. Ausiello and M. Lucertini, (eds.)., Springer-Verlag, New York, 1981, p. 147-172.

[21] Garey, M. and D. Johnson, A 71/60 theorem for bin packing, J. of Complexity, 1 (1985) 65-106.

[22] Graham, R., Bounds for certain multiprocessing anomalies, Bell System Tech. J., 45 (1966) 1563-1581.

[23] Graham, R., Bounds on multiprocessing anomalies, SIAM J. Applied Math., 17 (1969) 263-269.

[24] Graham, R., Combinatorial Scheduling, in Mathematics Today, L. Steen, (Ed.), Springer-Verlag, New York, 1978, p. 183-211

[25] Hofri, M., Probabilistic Analysis of Algorithms, Springer-Verlag, New York, 1987

[26] Johnson, D., Near-Optimal Bin Packing Algorithms, Doctoral Thesis, MIT, Cambridge, 1973.

[27] Average-Case Analyses of First Fit and Random Fit Bin Packing, Susanne Albers, Michael Mitzenmacher,

[28] Dósa G., Sgall J. (2013) First Fit bin packing: A tight analysis. To appear in STACS 2013

[29] D. Dutta, S.K.Jha, Seikh B. Ahmad, D.K.Pal, Implementation of Approximations Algorithms with Simulated Annealing (SA), IJARCSSE,2013 